\title{
DISTORTIONLESS WAVE PROPAGATION IN INHOMOGENEOUS MEDIA AND TRANSMISSION LINES*
}

\author{
$\mathrm{By}$ \\ V. BURKE (Case Western Reserve University), \\ R. J. DUFFIN (Carnegie-Mellon University), \\ AND \\ D. HAZONY (Case Western Reserve University),
}

\begin{abstract}
Of concern are mechanical or electrical waves in a media which may be nonuniform and dissipative. The problem posed is to find conditions for the undistorted propagation of signals. The electrical transmission line is chosen as the general model. Along the length of the transmission line there are four functions which may be prescribed essentially arbitrarily. These are series resistance, series inductance, shunt conductance, and shunt capacitance. A differential equation is derived relating these functions which gives a necessary and sufficient requisite for distortionless transmission of a voltage wave. Various corollaries of this theorem are developed. For instance, it is shown that simultaneous voltage and current waves can be transmitted without distortion if and only if the characteristic impedance of the transmission line is positive at each point.
\end{abstract}

1. Introduction. Distortionless signalling has been of fundamental importance in applied science. In this note we give a fairly general characterization of when this is possible. The simplest case of distortionless signalling arises in the equation of the vibrating string.

$$
V_{x x}=\omega^{2} V_{\iota}, \quad V_{x}=\partial V / \partial x, \quad V_{\iota}=\partial V / \partial t .
$$

This equation has the general solution

$$
V(t, x)=\phi(t-\omega x)+\psi(t+\omega x),
$$

where $\phi$ and $\psi$ are arbitrary functions. Thus $\phi(t-\omega x)$ is a wave traveling to the right with velocity $\omega^{-1}$. This solution is of great practical importance because this wave is transmitted to a distant point $x$ without distortion. There is, of course, a delay time $\tau=\omega x$.

The equation for spherical waves in $n$ dimensions is

$$
V_{r r}+\frac{n-1}{r} V_{r}=\omega^{2} V_{t t} .
$$

If $n=3$, the general solution of this equation is

$$
V(t, r)=\frac{\phi(t-\omega r)}{r}+\frac{\psi(t+\omega t)}{r} .
$$

* Received December 7, 1974. Work partially supported by Research Grant DA-ARO-D-31-124-73 G131, Army Research Office, Durham, North Carolina, and by National Science Foundation Grant GK 42720. 
Again it is seen that a wave can be transmitted without distortion but that there is an attenuation inversely proportional to the distance $r$. It is a very happy circumstance that we live in three-dimensional space because signals can be broadcast without distortion only if $n=1$ or $n=3$. This has been discussed by Courant [1].

Waves can be of mechanical or electrical nature. In this paper we take as a general model the electrical transmission line. The line can be inhomogeneous (nonuniform) and dissipative. An infinitesimal section of such a line is shown in Fig. 1 having series resistance $r$, series inductance $l$, shunt conductance $\ell$, and shunt capacitance $c$. Thus applying Kirchhoff's laws gives the transmission line equation:

$$
\begin{aligned}
-V_{x} & =r(x) I+l(x) I_{t}, \\
-I_{x} & =g(x) V+c(x) V_{t} .
\end{aligned}
$$

Here $V(t, x)$ is the voltage and $I(t, x)$ is the current. We proceed to operate on both sides of (1.5a) with $(r+(l \partial / \partial t)) \partial / \partial x$. The current is then eliminated by use of $(1.5 \mathrm{~b})$. This shows that the voltage satisfies:

$$
\left[\left(r+l \frac{\partial}{\partial t}\right) \frac{\partial^{2}}{\partial x^{2}}-\left(r^{\prime}+l^{\prime} \frac{\partial}{\partial t}\right) \frac{\partial}{\partial x}-\left(r+l \frac{\partial}{\partial t}\right)^{2}\left(g+c \frac{\partial}{\partial t}\right)\right] V=0 .
$$

This may be termed the general equation of telegraphy. The wave equations (1.1) and (1.3) are seen to be special cases of the equation of telegraphy.

We shall say that the transmission line is distortionless if, given an arbitrary function $\phi(t)$, there is a solution of the form

$$
V(t, x)=f(x) \phi[t-\tau(x)],
$$

with

$$
V(t, 0)=\phi(t) .
$$

Here the function $f$ is termed the attenuation and the function $\tau$ is termed the delay time. Of course $f$ and $\tau$ are to be independent of $\phi$. Also, $f(0)=1$ and $\tau(0)=0$.

Heaviside [2] originated the concept of distortionless transmission in a dissipative line. He proved that the condition for no distortion is

$$
r c-g l=0 \text {, }
$$

provided $r, c, g$ and $l$ are constant. Thus Heaviside solved the problem for a homogeneous line. An exponential line has the parameters $r, c, g$, and $l$ tapering exponentially. Recently

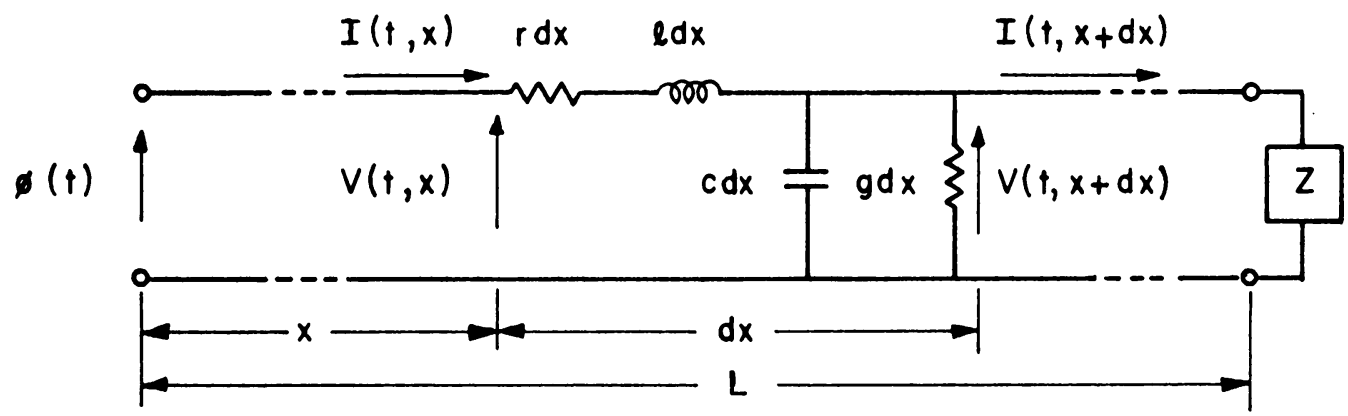

FIG. 1. Differential segment of a transmission line. 
Ghosh [3] has shown that the exponential line is distortionless if the tapering is suitably chosen.

Our goal is to characterize the general distortionless line. The above examples will then be special cases of our characterization. In what follows it is assumed that $l>0$ and $c>0$ for basic physical reasons. However, we do allow the idealizations $r=0$ and $g=0$. Furthermore, any attenuation function is assumed to be non-zero. Thus we are permitted to divide by $l, c$, and $\zeta=(l / c)^{1 / 2}$, and any attenuation function. Initially only waves traveling in the positive $x$ direction are considered. Thus $\tau(x)>0$ for $x>0$. This requires that the general line of finite length be terminated in the characteristic impedance $Z$ in order to eliminate any reflections from the end at $x=L$.

The functions $r, l, c$, and $g$ are assumed continuously differentiable throughout the paper.

We begin by treating the important special case of transmission lines whose characteristic impedance is purely resistive (Sec. 2). This means that $Z=V / I$ is a function only of $x$. The necessary and sufficient condition (Sec. 3) for a line to be resistive is that $\zeta=(l / c)^{1 / 2}$ solve the Riccati differential equation

$$
\zeta^{\prime}=g \zeta^{2}-r
$$

where a prime denotes $d / d x$. It is then found that any resistive line is distortionless and the delay time $\tau(x)$ satisfies

$$
\tau^{\prime}=(l c)^{1 / 2} .
$$

For plane waves in a vacuum it is well known that the electric and magnetic energy densities are equal. This equipartition property is shown to hold for signals on a transmission line provided the characteristic impedance is positive.

If the characteristic impedance is not resistive than it is found that the ratio $r / l$ must be constant if the line is to be distortionless (Sec. 4). Moreover, another Riccati differential equation, analogous to (1.9), must be satisfied.

In Sec. 7 the question of return signals is treated. Conditions are found which insure that distortionless signals can be transmitted to the left as well as to the right.

A great deal of study has been given to inhomogeneous lines by many authors. Some selected references relating to impedance transformation, filtering, signal dispersion and acoustics are given $[4,5,6,7,8]$.

2. Lines with voltage and current both distortionless. Consider the interesting special case when the transmission line is distortionless and when the ratio of voltage to current at any point of the line is a function only of $x$ and not of $t$. This means that in addition to

$$
V(t, x)=f(x) \phi[t-\tau(x)]
$$

we have

$$
I(t, x)=h(x) \phi[t-\tau(x)]
$$

for some function $h(x)$. Substitution of (2.1) into (1.5) gives

$$
\begin{aligned}
-f^{\prime} \phi+f \tau^{\prime} \phi_{t} & =r h \phi+l h \phi_{t}, \\
-h^{\prime} \phi+h \tau^{\prime} \phi_{t} & =g f \phi+c f \phi_{t} .
\end{aligned}
$$


There is no correlation between $\phi$ and $\phi_{t}$, so (2.1a) and (2.1b) hold iff

$$
\begin{aligned}
-f^{\prime} & =r h, \\
-h^{\prime} & =g f, \\
f \tau^{\prime} & =l h, \\
h \tau^{\prime} & =c f,
\end{aligned}
$$

Theorrm 2.1. The general transmission line is both voltage and current distortionless if and only if

$$
\begin{aligned}
\zeta^{\prime} & =g \zeta^{2}-r, \\
\tau & =\int_{0}^{x}(l c)^{1 / 2} d x, \\
f & =\exp \left[-\int_{0}^{x}(r / \zeta) d x\right], \\
h & =\left(1 / \zeta_{0}\right) \exp \left[-\int_{0}^{x} g \zeta d x\right],
\end{aligned}
$$

with $\zeta$ given $b y$

$$
\zeta=(l / c)^{1 / 2} .
$$

Proof. First suppose that relations (2.3) hold. Multiplying (2.3c) and (2.3d) gives

$$
\tau^{\prime}=(l c)^{1 / 2}
$$

which proves $(2.4 \mathrm{~b})$. Then $(2.3 \mathrm{c})$ gives

$$
f / h=l / \tau^{\prime}=(l / c)^{1 / 2}=\zeta .
$$

Thus $h(0)=f(0) / \zeta(0)=1 / \zeta_{0}$. Then relation (2.9) substituted into the right-hand side of $(2.3 \mathrm{a})$ and $(2.3 \mathrm{~b})$ gives

$$
\begin{aligned}
-f^{\prime} / f & =r / \zeta, \\
-h^{\prime} / h & =g \zeta,
\end{aligned}
$$

which proves relations (2.4c) and (2.4d). Eqs. (2.3b) and (2.3c) give $g f=-(f / \zeta)^{\prime}$ or gf $f=-f^{\prime} / \zeta+f \zeta^{\prime} / \zeta^{2}$; then (2.10a) results in (2.4a).

Conversely, assume relations (2.4) hold. Differentiate (2.4c) to give $f^{\prime}=(-r / \zeta) f$. Then $(2.4 \mathrm{~d})$ and $(2.4 \mathrm{a})$ give

$$
f^{\prime} / h=-(r / \zeta) \exp \left[\int_{0}^{x}\left(\zeta^{\prime} / \zeta\right) d x\right]=-r .
$$

This proves (2.3a). (2.3b) is proven similarly. Relations (2.4b), (2.4c), and (2.4d) give

$$
\tau^{\prime} f / h=(l c)^{1 / 2} \exp \left[\int_{0}^{x}(g \zeta-r / \zeta) d x\right]=l .
$$

This proves (2.3c). (2.3d) is obtained similarly. This completes the proof. 
3. Inductance, capacitance, and impedance of a line. There are standard procedures from potential theory for calculation of $c$, the capacitance per unit length of a transmission line. Also there are standard procedures from potential theory for calculating $l$, the inductance per unit length. For parallel wires in a vacuum this well-known calculation gives that $1 /(l c)^{1 / 2}$ is equal to the velocity of light. Likewise for a coaxial cable, $1 /(l c)^{1 / 2}$ is the velocity of light. The question then arises whether or not $l c$ is a constant independent of the cross-section of the transmission line. In previous papers $[9,10]$, it was shown by use of Rayleigh's reciprocal theorem that in a vacuum $l c$ is an invariant. No use of Maxwell's theory was made to establish this fact.

By inserting dielectric material between the conductors of the transmission line it is possible to increase $c$. By inserting magnetically permeable material it is possible to increase $l$. In such ways it is possible to have $l c$ be an arbritary function along the line subject to the restriction

$$
0<1 /(l c)^{1 / 2} \leq \text { velocity of light. }
$$

The upper bound here would follow from the special theory of relativity.

Let us seek solutions of the transmission line equations which are sinusoidal in time, say

$$
I=p(x) \exp (s t), \quad V=q(x) \exp (s t) \not \equiv 0 .
$$

Here $s=2 \pi i v$ and $\nu$ is the frequency. Then Eqs. (1.5a) and (1.5b) become

$$
\begin{aligned}
& -d q / d x=(r+l s) p, \\
& -d p / d x=(g+c s) q .
\end{aligned}
$$

The characteristic impedance function $Z(x)$ is defined by

$$
q(x)=Z(x) p(x) .
$$

LEmma 3.1. The characteristic impedance function of the general transmission line satisfies the Riccati differential equation

$$
d Z / d x=[g(x)+s c(x)] Z^{2}-[r(x)+s l(x)],
$$

except possibly at isolated points.

Proof. Use relation (3.3) to eliminate $q$ in (3.2). Thus

$$
-Z p_{\iota}-Z_{x} p=(r+l s) p, \quad-p_{x}=(g+c s) Z p .
$$

Elimination of $p$ between these last two equations gives (3.4) if $p \neq 0$. If $p$ were to vanish in an interval then Eq. (3.3) shows that $q$ would also vanish. Then Eq. (3.2) would imply that $p$ and $q$ vanish everywhere and this contradicts the hypothesis (3.1). This completes the proof.

TheOREm 3.1. A transmission line has a positive characteristic impedance function $Z(x)$ if and only if

$$
d \zeta / d x=g(x) \zeta^{2}-r(x), \quad \zeta=(l / c)^{1 / 2} .
$$

Moreover, $Z$ is uniquely defined as

$$
Z=\zeta
$$


Proof. If $Z(x)$ is given to be positive then setting the real and imaginary parts of Eq. (3.4) equal to zero gives Eqs. (3.5) and (3.6). On the other hand, if relation (3.5) is satisfied we know from Theorem 2.1 that $V=f(x) \phi[t-\tau(x)]$ and $V=\zeta I$. Choose $\phi(t)=\exp (s t)$ and it is seen that the impedance is $\zeta$ and so is positive.

Corollary 3.1. A transmission line is both voltage- and current-distortionless if and only if it has a positive characteristic impedance function.

4. The general distortionless line. We now require that the voltage $V$ be distortionfree. We do not require that $I$ be distortion-free. Again, let

$$
V(t, x)=f(x) \phi[t-\tau(x)] .
$$

The following voltage derivatives are obtained from (4.1).

$$
\begin{aligned}
V_{x} & =f^{\prime} \phi-f \tau^{\prime} \phi_{t}, \\
V_{x x} & =f^{\prime \prime} \phi-\left(2 f^{\prime} \tau^{\prime}+f \tau^{\prime \prime}\right) \phi_{t}+f\left(\tau^{\prime}\right)^{2} \phi_{t \imath},
\end{aligned}
$$

Substituting into the equation of telegraphy (1.6) gives

$$
\begin{gathered}
{\left[l\left(\tau^{\prime}\right)^{2}-l^{2} c\right] \phi_{l t t}+[-} \\
\left.-2 l \tau^{\prime} f^{\prime}-\left(2 r c l-r\left(\tau^{\prime}\right)^{2}+l^{2} g+l \tau^{\prime \prime}-l^{\prime} \tau^{\prime}\right) f\right] \phi_{t \iota} \\
+\left[l f^{\prime \prime}-\left(2 r \tau^{\prime}+l^{\prime}\right) f^{\prime}-\left(r \tau^{\prime \prime}-r^{\prime} \tau^{\prime}+2 r g l+r^{2} c\right) f\right] \phi_{\iota} \\
+\left[r f^{\prime \prime}-r^{\prime} f^{\prime}-r^{2} g f\right] \phi=0 .
\end{gathered}
$$

Since $\phi$ is an arbitrary signal the coefficients of $\phi_{l u}, \phi_{l l}, \phi_{l}$, and $\phi$ must all vanish. This proves the following:

Lemma 4.1. The general transmission line is voltage-distortionless if and only if there is a function $f(x)$, not identically zero, satisfying the differential equations:

$$
\begin{aligned}
-2 l \tau^{\prime} f^{\prime} & =\left(2 r c l-r\left(\tau^{\prime}\right)^{2}+l^{2} g+l \tau^{\prime \prime}-l^{\prime} \tau^{\prime}\right) f, \\
l f^{\prime \prime}-\left(2 r \tau^{\prime}+l^{\prime}\right) f^{\prime} & =\left(r \tau^{\prime \prime}-r^{\prime} \tau^{\prime}+2 r g l+r^{2} c\right) f, \\
r f^{\prime \prime} & =r^{\prime} f^{\prime}+r^{2} g f,
\end{aligned}
$$

and the delay time is given by

$$
\tau^{\prime}=(l c)^{1 / 2} .
$$

This leads to the following:

Theorem 4.1. A general transmission line is voltage-distortionless if and only if the delay time is given by

$$
\tau^{\prime}=(l c)^{1 / 2}
$$

and the function $H$, defined as

$$
H=\left(g \zeta-r / \zeta-\zeta^{\prime} / \zeta\right) / 2
$$

satisfies the relations

$$
H^{\prime}=H^{2}+\left(l^{\prime} / l\right) H
$$

and

$$
(r / l)^{\prime} H=0 .
$$


Proof. First assume relations (4.4) through (4.7) hold. Then by Lemma 4.1 the line is distortionless. Let the functions $f$ and $H$ be related as

$$
-f^{\prime}=(H+r / \zeta) f
$$

Divide (4.4) by $2 l \tau^{\prime} f$. Then from (4.7), (4.11), and the identity

$$
l^{\prime} / l-\tau^{\prime \prime} / \tau^{\prime}=\zeta^{\prime} / \zeta
$$

relation (4.8) is obtained. From (4.11),

$$
\left.f^{\prime \prime}=\left[(H+r / \zeta)^{2}-(H+r / \zeta)^{\prime}\right]\right] .
$$

Divide (4.5) by $l$ and employ (4.11) and (4.13) to eliminate $f^{\prime}$ and $f^{\prime \prime}$. Collect terms in $H$ to one side giving $H^{2}-H^{\prime}+\left(l^{\prime} / l\right) H+4(r / \zeta) H$. Employ $\zeta=(l / c)^{1 / 2}, \tau^{\prime}=(l c)^{1 / 2}$, and (4.12) to reduce the remaining terms to $2(r / \zeta)\left(g \zeta-r / \zeta-\zeta^{\prime} / \zeta\right)$ which, by (4.8), equals $4(r / \zeta) H$. This proves (4.9). To prove (4.10), eliminate $f^{\prime \prime}$ and $f^{\prime}$ in (4.6) to give

$$
r H^{\prime}=r H^{2}+r^{\prime} H .
$$

Subtract $r$ times (4.9) from (4.14) to obtain (4.10).

Conversely, assume relations (4.7) through (4.10) hold. Substitute (4.8) into (4.11) and eliminate $\zeta^{\prime} / \zeta$ with (4.12), giving

$$
-f^{\prime}=\left(r / \zeta+g \zeta+\tau^{\prime \prime} / \tau^{\prime}-l^{\prime} / l\right) f / 2 .
$$

Then multiply by $2 l \tau^{\prime}$ and employ $\zeta=(l / c)^{1 / 2}$ and $\tau^{\prime}=(l c)^{1 / 2}$ to give (4.4). Relation (4.5) is obtained as follows. Subtract $\left[2(r / \zeta)+\left(l^{\prime} / l\right)\right] f^{\prime}$ from both sides of (4.13). Then simplify the right side with (4.9) and (4.11), giving

$$
f^{\prime \prime}-\left[2(r / \zeta)+\left(l^{\prime} / l\right)\right] f^{\prime}=\left[4(r / \zeta) H+3(r / \zeta)^{2}+(r / \zeta)\left(l^{\prime} / l\right)-(r / \zeta)^{\prime}\right] f .
$$

Then (4.8) and (4.12) give [2rg $\left.+(r / \zeta)^{2}+(r / \zeta)\left(\tau^{\prime \prime} / \tau^{\prime}\right)-r^{\prime} / \zeta\right]$ on the right. Multiply by $l$ and employ $\zeta=(l / c)^{1 / 2}$ and $\tau^{\prime}=(l c)^{1 / 2}$ to give (4.5). To prove (4.6), expand (4.13), eliminating $H^{\prime}$ with (4.9) and $2 H r / \zeta$ with (4.8), giving

$$
r f^{\prime \prime}=r\left[r g-\left(r^{\prime} / \zeta\right)-\left(l^{\prime} / l\right) H\right] f .
$$

Add $-r^{\prime} f^{\prime}=r^{\prime}[H+(r / \zeta)] f$ to both sides, giving

$$
r f^{\prime \prime}-r^{\prime} f^{\prime}=\left[r^{2} g+\left(r^{\prime}-r l^{\prime} / l\right) H\right] f .
$$

Then (4.10) gives (4.6). Thus Lemma 4.1 is satisfied and the theorem is proved.

Corollary 4.1. If $r / l$ is not constant on any interval the line is distortionless if and only if the characteristic impedance function is positive as in Theorem 2.1.

Proof. Eq. (4.10) is true for any distortionless line. By the hypothesis of the corollary, $(r / l)^{\prime}$ can only vanish at isolated points. Thus by continuity

$$
H=0 .
$$

Thus employing the relation for $H$ in (4.8) yields

$$
g \zeta-r / \zeta-\zeta^{\prime} / \zeta=0
$$

However, this is the condition of Theorem 2.1 which insures that the characteristic impedance is positive and Corollary 4.1 follows. 
Corollary 4.2. If $r / l$ is constant the line is distortionless if and only if

$$
H^{\prime}=H^{2}+\left(l^{\prime} / l\right) H
$$

where

$$
H=1 / 2\left(g \zeta-r / \zeta-\zeta^{\prime} / \zeta\right)
$$

Furthermore, the attenuation factor is

$$
f(x)=\exp \left[-\int_{0}^{x}(H+r / \zeta) d x\right]
$$

the delay is

$$
\tau(x)=\int_{0}^{x}(l c)^{1 / 2} d x
$$

and the characteristic impedance function is

$$
Z=\frac{s+r / l}{s+(H+r / \zeta) / \tau^{\prime}} \zeta
$$

where $s=2 \pi_{i} \nu$ and $\nu$ is the frequency.

Proof. From the hypothesis $(r / l)^{\prime}=0$. Thus (4.10) is satisfied identically. By Theorem 4.1 and Lemma 4.1 the line is distortionless iff (4.17) and (4.18) are satisfied. Eq. (4.11) proves (4.19) while (4.7) proves (4.20). Relation (4.21) is proved as follows. Assume $\phi(t)$ has a sinusoidal time dependence

$$
\phi(t)=\exp (s t),
$$

where $s=2 \pi i \nu$ and $\nu$ is the frequency. Then, from (4.1),

$$
V=f(x) \exp (s[t-\tau(x)]) .
$$

The current also has a sinusoidal time dependence. Substituting (4.23) into (1.5a) gives

$$
\left(-f^{\prime} / f+s \tau^{\prime}\right) V=(r+s l) I .
$$

This result and (3.1) give

$$
\left(-f^{\prime} / f+s \tau^{\prime}\right) q(x)=(r+s l) p(x) .
$$

Thus from (4.25), (3.3), and (4.11) relation (4.21) is obtained. This completes the proof.

5. Equipartion of electric and magnetic energy. For the classical wave equation it is known that there is a tendency for the potential energy and the kinetic energy to become equal as time goes on. In odd-dimensional space this equipartition tendency is stronger and the following property holds. If initially a wave has finite support then after a sufficient time has elapsed the potential energy equals the kinetic energy and remains equal for all later times [11].

We wish to point out a related equipartition of energy property for the transmission line. As usual, electric field energy is to be interpreted as potential energy and magnetic field energy is to be interpreted as kinetic energy.

THEOREM 5.1. If a line has a positive characteristic impedance function then the electric energy density and the magnetic energy density of a signal are equal. 
Proof. Of course the electric energy density is $c(x) V^{2}(t, x) / 2$ and the magnetic energy density is $l(x) I^{2}(t . x) / 2$. Because of Corollary 3.1 we know that Theorem 2.1 applies. Thus $V=\zeta I$ so $c V^{2}=c \zeta^{2} I^{2}=c(l / c) I^{2}=l I^{2}$.

6. Return signals. Up to this point we have been concerned only with transmission of signals to the right. Now we treat transnission lines which permit distortionless signalling both to the right and to the left. First it is convenient to restate Theorem 4.1 as

Theorem 6.1. A transmission line is right-distortionless if and only if

$$
\begin{gathered}
H^{\prime}=H^{2}+\left(l^{\prime} / l\right) H, \\
(r / l)^{\prime} H=0,
\end{gathered}
$$

where

$$
H=\left(g \zeta-r / \zeta-\zeta^{\prime} / \zeta\right) / 2 .
$$

The corresponding theorem for distortionless signalling to the left is

TheOREM 6.2. A transmission line is left-distortionless if and only if

$$
\begin{gathered}
\tilde{H}^{\prime}=\tilde{H}^{2}+\left(l^{\prime} / l\right) \tilde{H}, \\
(r / l)^{\prime} \tilde{H}=0,
\end{gathered}
$$

where

$$
\tilde{H}=\left(-g \zeta+r / \zeta-\zeta^{\prime} / \zeta\right) / 2 .
$$

Proof. Theorem 6.2 is derived from Theorem 6.1 by making the transformation $d x \rightarrow-d x$. Under this transformation $H \rightarrow-\tilde{H}$ and relations (6.4) and (6.5) are seen to follow.

Suppose that a transmission line is both left- and right-distortionless. Then relations (6.1) and (6.4) both hold. Subtracting these relations gives

$$
(\tilde{H}-H)^{\prime}=(\tilde{H}+H)(\tilde{H}-H)+\left(l^{\prime} / l\right)(\tilde{H}-H) .
$$

Let

$$
\beta=r / \zeta-g \zeta,
$$

so $\tilde{H}-H=\beta$ and (6.7) becomes

$$
\beta^{\prime}=-\left(\zeta^{\prime} / \zeta\right) \beta+\left(l^{\prime} / l\right) \beta .
$$

Integration of (6.9) gives $\beta \zeta / l=$ const. But $\zeta=(l / c)^{1 / 2}$ so this relation becomes

$$
(r / l)-(g / c)=\text { const. }
$$

Corollary 6.1. A transmission line is left-and right-distortionless if:

$$
\begin{gathered}
H^{\prime}=H^{2}+\left(l^{\prime} / l\right) H, \\
(r / l)^{\prime}=0, \\
(g / c)^{\prime}=0 .
\end{gathered}
$$

Proof. Relations (6.11) and (6.12) insure that the line is right-distortionless. Relations (6.12) and (6.13) insure that relation (6.10) holds. But (6.10) implies (6.9), 
which implies (6.7). It is now evident that the conditions (6.4) and (6.5) of Theorem 6.2 hold and so the line is left-distortionless.

Corollary 6.2. A transmission line is left-and right-distortionless both for voltage and current if and only if

$$
\begin{aligned}
l / c & =\text { constant } \\
r & =(l / c) g .
\end{aligned}
$$

Proof. We have $\tilde{H}+H=-\zeta^{\prime} / \zeta$. Thus (6.14) is equivalent to $\tilde{H}+H=0$. Likewise $\tilde{H}-H=r / \zeta-g \zeta$ and so Eq. (6.15) is equivalent to $\tilde{H}-H=0$. This shows that (6.14) and (6.15) are equivalent to $\tilde{H}=H=0$. Then Theorem 2.1 completes the proof of the corollary. Also, Theorem 3.1 shows that the characteristic impedance function has the constant value $(l / c)^{1 / 2}$.

7. Applications. Several examples of distortionless lines will be discussed and results summarized in Table I. We begin the illustrations by discussing a line whose voltage propagation is analogous to the solution of the three-dimensional spherical wave equation discussed in Sec. 1. Let $r=g=0$ and $l=c^{-1}=(x+1)^{-2}$. Since $r / l=0$, Corollary 4.2 applies. Relation (4.18) gives

$$
H=1 /(x+1)^{-1},
$$

while (4.17) gives

$$
H^{\prime}=H^{2}-[2 /(x+1)] H .
$$

TABLE 1.

\begin{tabular}{|c|c|c|c|}
\hline & $\begin{array}{c}\text { Spherical Analogy } \\
(r=g \equiv 0)\end{array}$ & $\begin{array}{c}\text { Generalized Exponential (GE) } \\
\left(\beta_{0} \Delta r_{0} / \zeta_{0}-g_{0} \zeta_{0} ; A \text { arbitrary }\right)\end{array}$ & $\begin{array}{c}\text { Unidirectional } \\
\left(r_{0}=\zeta_{0}\right)\end{array}$ \\
\hline$l$ & $l_{0}(x+1)^{-2}$ & $\frac{(1+A)^{2}}{\left(e^{\beta_{0} x / 2}+A e^{-\beta_{0} x / 2}\right)^{2}} l_{0}$ & $l_{0}$ \\
\hline$c$ & $c_{0}(x+1)^{2}$ & $\frac{\left(e^{\beta_{0} x / 2}+A e^{-\beta_{0} x / 2}\right)^{2}}{(1+A)^{2}} c_{0}$ & $c_{0} e^{2 x}$ \\
\hline$r$ & 0 & $\frac{(1+A)^{2}}{\left(e^{\beta_{0} x / 2}+A e^{-\beta_{0} x / 2}\right)} r_{0}$ & $r_{0} e^{-x}$ \\
\hline$g$ & 0 & $\frac{\left(e^{\beta_{0} x / 2}+A e^{-\beta_{0} x / 2}\right)^{2}}{(1+A)^{2}} g_{0}$ & 0 \\
\hline$Z$ & $s$ & $s+\frac{1}{2}\left(\frac{r_{0}}{l_{0}}+\frac{g_{0}}{c_{0}}\right)+\frac{\beta_{0} / 2}{\sqrt{l_{0} c_{0}}} e^{\beta_{0} x / 2}-A e^{-\beta_{0} x / 2}$ & $\zeta_{0} e^{-x}$ \\
\hline$s+1 / \sqrt{l_{0} c_{0}}(x+1)$ & $\frac{(1+A)}{e^{-\beta_{0} x / 2}}$ & \\
\hline$f$ & $(x+1)^{-1}$ & $\sqrt{\beta_{0} x / 2}+A e^{-\beta_{0} x / 2} e^{-1 / 2\left(c_{0} \zeta_{0}+r_{0} / \zeta_{0}\right) x}$ & $e^{-x}$ \\
\hline$\tau$ & $\sqrt{l_{0} c_{0} x}$ & $\sqrt{l_{0} c_{0} x}$ & $\sqrt{l_{0} c_{0}}\left(e^{x}-1\right)$ \\
\hline
\end{tabular}


$H$ given by (7.1) is a solution of (7.2), thus the line is distortionless. From Eq. (4.19), $f=1 /(x+1)$.

The generalized exponential line is defined by the following relations among the parameters:

$$
\mathbf{r}=1=\mathbf{c}^{-1}=\mathbf{g}^{-1}=\exp \left[-\int_{0}^{x} \alpha(x) d x\right],
$$

where $\mathbf{r}=r / r_{0}, r(0)=r_{0}$ and similarly for $\mathbf{1}, \mathbf{c}$, and $\mathbf{g}$. Here $\alpha(x)$ is a function which characterizes the taper.

Corollary 7.1. A generalized exponential line is voltage distortionless if $\alpha(x)$ satisfies

$$
\left(\alpha^{\prime} / 2\right)+(\alpha / 2)^{2}=\left(\beta_{0} / 2\right)^{2},
$$

with

$$
\beta_{0}=r_{0} / \zeta_{0}-g_{0} \zeta_{0} .
$$

Proof. By hypothesis $r / l=$ const. so Corollary 4.2 applies. Relations (4.18), (7.3), and (7.5) give

$$
H=\alpha / 2-\beta_{0} / 2
$$

while (4.17) may be written

$$
H^{\prime}=H^{2}-\alpha H .
$$

$H$ can be eliminated by inserting (7.6) in (7.7). This results in (7.4). Thus when (7.4) is satisfied so is Corollary 4.2 and the proof is complete.

Eq. (7.4) is integrable and has the general solution

$$
\alpha(x)=\beta_{0} \frac{\exp \left(\beta_{0} x / 2\right)-A \exp \left(-\beta_{0} x / 2\right)}{\exp \left(\beta_{0} x / 2\right)+A \exp \left(-\beta_{0} x / 2\right)},
$$

where $A$ is an arbitrary real number. The following special solutions are of interest:

(a) Exponential line.

(i) For $A=0, \alpha=\beta_{0}$, a constant. Then (7.3) gives

$$
1=\mathrm{r}=\mathrm{g}^{-1}=\mathrm{c}^{-1}=\exp \left(-\beta_{0} x\right) .
$$

Furthermore the parameters of (7.9) satisfy Eq. (2.4) of Theorem 2.1 so with $\alpha=\beta_{0}$ both the voltage and the current are distortionless.

(ii) For $A=\infty, \alpha=-\beta_{0}$. Thus

$$
1=\mathrm{r}=\mathrm{g}^{-1}=\mathrm{c}^{-1}=\exp \left(\beta_{0} x\right) .
$$

The exponential line with $\alpha=-\beta_{0}$ has the impedance

$$
Z=\frac{s+r_{0} / l_{0}}{s+\left(r_{0} / l_{0}+g_{0} / c_{0}\right)} \zeta_{0} \exp \left(\beta_{1} x\right),
$$

which follows from (4.21) and (4.18).

(iii) Heaviside line. The exponential line reduces to a uniform line when $\beta_{0}=0$. 
In this case (7.5) gives $r_{v} c_{0}-g_{0} l_{0}=0$, which is the Heaviside condition for distortionless transmission in a uniform line.

(b) Hyperbolic lines.

(i) For $A=+1,(7.8)$ gives

$$
\alpha(x)=\beta_{0} \tanh \left(\beta_{0} x / 2\right) .
$$

Consequently, the parameters are

$$
1=\mathbf{r}=\mathbf{g}^{-1}=\mathbf{c}^{-1}=1 / \cosh ^{2}\left(\beta_{0} x / 2\right) .
$$

(ii) For $A=-\exp \left(-\beta_{0} a\right)$, (7.8) gives

$$
\alpha(x)=\beta_{0} \operatorname{coth}\left[\beta_{0}(x+a) / 2\right] \quad(a>0) .
$$

Then the parameters are

$$
1=\mathbf{r}=\mathbf{g}^{-1}=\mathbf{c}^{-1}=\sinh ^{2}\left(\beta_{0} a / 2\right) / \sinh ^{2}\left[\beta_{0}(x+a) / 2\right] .
$$

Unidirectional lines. Let $g=0, l=$ unity, $r=\zeta=\exp (-x)$. It is seen that $\zeta^{\prime}=$ $g \zeta^{2}-r$ and therefore voltage-distortionless waves are permitted. Suppose now that at the point $x$ a signal is sent in the opposite direction. Thus $d x \rightarrow-d x$ and

$$
\zeta^{\prime}=-(d / d x) \exp (-x)=\exp (-x) \text {. }
$$

Now $\zeta^{\prime} \neq g \zeta^{2}-r$. Moreover, $(r / l)^{\prime} \neq 0$, showing that Corollary 4.2 does not apply and hence no voltage-distortionless waves to the left are possible.

Lines with the product $l c=$ constant are characterized by a linear delay. This follows from relation (4.7). In addition to the unidirectional property, the last example has a nonlinear delay time $\tau=\left(l_{0} c_{0}\right)^{1 / 2}(\exp [x]-1)$.

\section{REFERENCES}

[1] R. Courant, Hyperbotic partial differential equations and appiications, in E. F. Backenbach (ed.), Modern mathematics for the engineer, McGraw-Hill, 1956, pp. 92-109

[2] O. Heaviside, Electrical papers, Electrician 2, 125 (1887)

[3] S. K. Ghosh, Responses in a non-uniform electrical transmission iine, J. Inst. Eng. (India), Erec. Eng. Div., 51, 329-330 (1971)

[4] C. R. Burrows, The exponential transmission line, Bell System Technical Journal 17, 5i5;-5753 (1938)

[5] F. Bolinder, Fourier transforms in the theory of inhomogeneous transmission lines, Proc. IRE, 38, 1354 (1950)

[6] R. E. Collin, The optimum tapered transmission line matching section, Proc. IRE 44, 539-548 (1956)

[7] Staff, Bell Telephone Labs., Transmission systems for communications, Bell Telephone Labs., 1964

[8] O. K. Mawardi, Generalized solutions of Webster's horn theory, J. Acous. Soc. Amer. 21, 323-330 (1949)

[9] R. J. Iuffin, Distributed and lumped networks, J. Math. Mech. 8, 793-826 (1959)

[10] R. J. Duffin, The extremal length of a network, J. Math. Anal. Appl. 5, 200-215 (1962)

[11] R. J. Iuffin, Equipartition of energy in wave motion, J. Math. Anal. Appl. 32, 386-391 (1970) 